\title{
New Contact Development for Si(Li) Orthogonal-Strip Detectors
}

\author{
I. D. Hau ${ }^{\mathrm{a}, \mathrm{b}}$, C. Tindall, ${ }^{\mathrm{a},}$, P. N. Luke ${ }^{\mathrm{a}}$ \\ ${ }^{a}$ Lawrence Berkeley National Laboratory, Berkeley, CA 94720 \\ ${ }^{b}$ Department of Nuclear Engineering, University of California, Berkeley, CA 94720
}

\begin{abstract}
At present, the contacts generally used for lithium-drifted silicon detectors consist of a diffused lithium layer (n-type) and a gold surface barrier (p-type). These contacts work well for unsegmented detectors. However, they both have disadvantages if used for segmented detectors. For this reason, we are developing new types of contacts that will be more robust and easier to segment. To replace the lithium n-type contact, we are using a thin layer of amorphous silicon $(\alpha-\mathrm{Si})$ with metalization on top. The new p-type contact consists of boron implanted into the silicon and annealed at the relatively low temperature of $500{ }^{\circ} \mathrm{C}$. The implantation and annealing is carried out as the first step in the process, prior to lithium drifting. Detectors have been fabricated using the new contacts both with and without a guard ring. They performed as well as detectors with standard contacts at operating temperatures between $80 \mathrm{~K}$ and $240 \mathrm{~K}$. We will present data on the leakage current vs. temperature, isolation resistance between the guard ring and the center contact versus temperature and bias voltage, electronic noise and energy resolution versus temperature, as well as ${ }^{57}$ Co spectra.
\end{abstract}

Keywords: lithium drift silicon, amorphous silicon contact, boron implanted contact, thermal annealing, gamma-ray detector, orthogonal-strip detector.

\section{Introduction}

Lithium drifted silicon $(\mathrm{Si}(\mathrm{Li}))$ orthogonal-strip detectors are being developed at Lawrence Berkeley National Laboratory (LBNL) in order to be used in Compton telescopes. In such applications, the detection elements will consist of a three dimensional array of $\mathrm{Si}(\mathrm{Li})$ orthogonal-strip detectors and the detection technique will be based on Compton scattering. A reconstruction algorithm based on locating the first three Compton interactions in a low $\mathrm{Z}$ material detector is intended to be used to reconstruct the energy and the direction of the incident gamma ray photons [1]. In order to accurately use this detection algorithm, the individual detectors in the telescope must have good position resolution ( $\leq 2 \mathrm{~mm}$ ) and low noise ( $\leq 2 \mathrm{keV}$ FWHM). The additional requirement for a high operating temperature ( $200 \mathrm{~K}$ to $240 \mathrm{~K}$ ) makes it necessary to use silicon detectors instead of germanium since germanium requires liquid nitrogen cooling. The orthogonal strips on the front and back sides of the individual detector will be used to determine the $\mathrm{x}$ - and $\mathrm{y}$-coordinates of the Compton interaction, whereas the $\mathrm{z}$-coordinate will be determined based on a timing method ([2] and [3]).

One of the problems which has to be solved for $\mathrm{Si}(\mathrm{Li})$ detectors in order to create strip (segmented) detectors with a pitch on the order of $1 \mathrm{~mm}$ is the development of improved p-type and n-type contacts. Each type of contact that is presently used in conventional $\mathrm{Si}(\mathrm{Li})$ has disadvantages for use in segmented detectors.

\footnotetext{
* Corresponding author. Tel.: +1(510)486-6523 ; fax: +1(510)486-5857 e-mail: CSTindall@lbl.gov
}

The lithium contact (n-type) has a thickness between 20 microns and several hundred microns.

In order to create the segments, a significant amount of material must be removed either through chemical etching or mechanical means in order to isolate the segments from each other. From a practical standpoint, this is a potentially viable process, but this tends to limit the pitch resolution that can be obtained and there is a risk of electrical shorts between segments due to lithium migration. In the case of the gold surface barrier (p-type), the adhesion of the gold to the silicon is very poor, the contact is very fragile and the wire bonding to it is not possible.

\section{Detector fabrication and experimental arrangement}

For the development of the new n-type contact, conventional $\mathrm{Si}(\mathrm{Li})$ detectors were first fabricated using the standard LBNL process which has been described previously in [4] and [5]. The lithium contacts were then removed by lapping. The new n-type contact was then applied to the detectors, replacing the conventional contacts. For the development of the new p-type contact, the p-type contact formation was the first step in the detector fabrication process followed by the standard procedure of $\mathrm{Si}(\mathrm{Li})$ detector fabrication. Tests were performed on detectors with an overall diameter of 24 $\mathrm{mm}$ and thicknesses ranging from $2 \mathrm{~mm}$ to $5 \mathrm{~mm}$. Each detector has a deep circular groove cut into the silicon to define the active area, which has a diameter of $16.5 \mathrm{~mm}$ (Figure 1). No surface passivation treatment is applied to the groove.

For the tests reported here, guard ring detectors were used. In a guard ring detector, the active area is divided into an outer annular guard-ring $(\sim 1.5 \mathrm{~mm}$ wide) 
and a circular inner region (center contact). A gap of about $1.5 \mathrm{~mm}$ formed between the guard ring and the center contact separates the two regions. The gap is formed either by a masked deposition of the electric contacts or by etching away the annular region between the guard ring and the center contact. The purpose of the guard ring configuration is to test the contact segmentation process and to determine the electrical performance of the contacts without interference from surface leakage currents. The area of the center contact is comparable to the anticipated area of a single strip contact in the orthogonal-strip detector design, so that the test results obtained in this study can be directly translated to final detector performance.

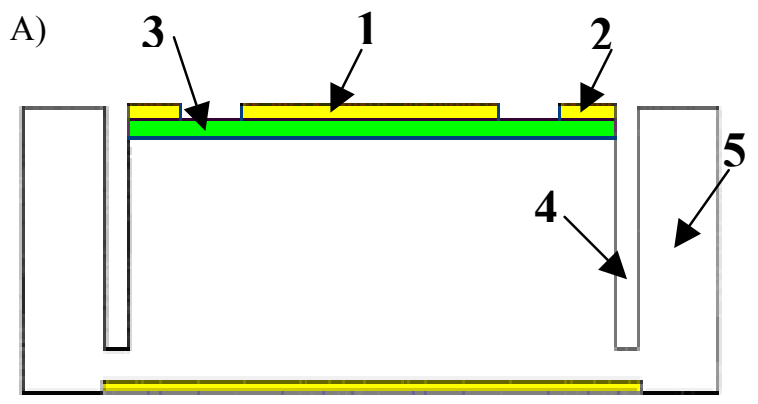

B)

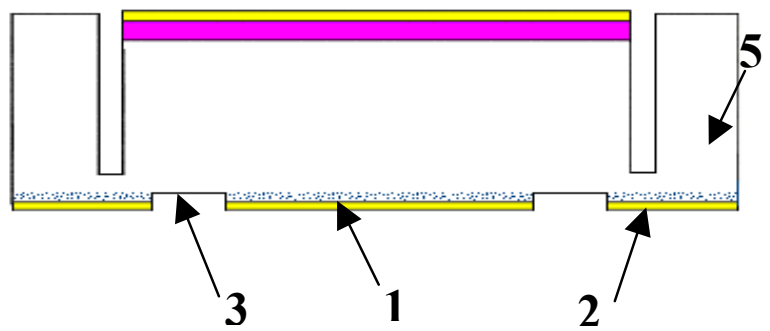

Figure 1. Cross section of a guard ring detector. A) N-type contact experiment, $5 \mathrm{~mm}$ thick detector B) P-type contact experiment, $2 \mathrm{~mm}$ thick detector. 1 - center contact, 2 - guard ring, 3 - shallow groove (gap), 4 - deep groove, 5 - passive material

The detectors were tested in a liquid nitrogen cooled cryostat. The temperature of the cold stage may be varied from $77 \mathrm{~K}$ to $396 \mathrm{~K}$. We have measured leakage current, detector noise, FWHM of gamma-ray lines, and isolation resistance between the guard ring and the center contact. The detector signal was collected using an LBNL custom-built low noise pre-amp. The electronic noise of the preamp (at $6.7 \mu$ s peaking time, no detector attached) is approximately $1.1 \mathrm{keV}$ FWHM. For the noise measurement the output is fed into a shaping amplifier followed by a true RMS voltmeter. Alternately, noise levels are determined by measuring the FWHM of pulser peaks obtained together with the gamma-ray spectra. The leakage current measurements were made with a pico ammeter. Except for contact isolation measurements, all measurements using the guard ring detectors are made with the guard ring grounded. The bias voltage was applied to the $\mathrm{Au}$ surface barrier contact for n-type contact experiments and on the lithium contact for the ptype contact experiments. To measure contact isolation, the resistance between contact elements in the guard ring detectors is computed based on the I-V characteristic measured between the guard ring and the center contact.

\section{Development of new n-type Contact}

In the process of developing new n-type contacts we performed experiments with both full area detectors and guard ring detectors in which the lithium contact is replaced by an amorphous-silicon $(\alpha-\mathrm{Si})$ contact ([6] and [7]). An $\alpha$-Si contact consists of an $\alpha$-Si layer deposited on the detector plus a layer of metal on top of the $\alpha$-Si to define a low-resistance contact area. Using $\alpha$-Si as an ntype contact is made possible by the fact that the interface between $\alpha$-Si and the metal contact evaporated in top of it seems to have an ohmic nature, whereas the $\alpha-\mathrm{Si}-$ crystalline Si interface behaved like a Schottky contact [7]. This is due to the fact that the Fermi level of $\alpha$-Si lies somewhere within the band gap of crystalline silicon. Previous experiments showed that layers of $\alpha-G e$ and $\alpha$-Si worked well as contacts for germanium detectors and the segmentation was relatively simple [2].

In order to test the $\alpha$-Si contact, we started with conventional $6 \mathrm{~mm}$ thick $\mathrm{Si}(\mathrm{Li})$ detectors. The lithium contact was removed by grinding away the top $1 \mathrm{~mm}$ of the detector. The surface was then etched and sputter coated with a layer of $\sim 300$ Angstroms of $\alpha$-Si followed by a layer of $\sim 400$ Angstroms of aluminum. We have experimented with $\alpha$-Si sputtered in pure argon and argon mixed with $17.5 \%$ hydrogen, and found that detectors made with the latter process give substantially better performance. Therefore, results presented here are for contacts made by sputtering in argon with 17.5\% hydrogen. Detector 8911 was fabricated in full area configuration using the $\alpha$-Si contact. After testing, a guard ring structure was made by using a masking process. In this process, the initial aluminum layer on top of the $\alpha-\mathrm{Si}$ was removed. The gap separating the guard ring and the center contact of the detector was covered by a ring-shaped shadow mask, then a new layer of 400 Angstroms of aluminum was evaporated on top on the $\alpha$ Si layer. The metalized areas serve to define the guard ring and the center contact. The p-type contact of detector 8911 consists of a standard $\mathrm{Au}$ surface barrier. The window thickness of the resulting $\alpha-\mathrm{Si}$ contact is calculated as being of about 0.1 micron, based on measuring the peak energy from the spectrum of an ${ }^{241} \mathrm{Am}$ alpha-particle source at ambient temperature. We measured the leakage current, the FWHM of the $122 \mathrm{keV}$ peak obtained from ${ }^{57} \mathrm{Co}$ spectra, and calculate the isolation resistance between the guard ring and the center contact. For the purpose of developing contacts for $\mathrm{Si}(\mathrm{Li})$ detectors operable at temperatures higher than $80 \mathrm{~K}$, we 
are especially interested in the temperature range from $200 \mathrm{~K}$ to $240 \mathrm{~K}$, which would reduce cooling requirement sufficiently to be practical for large systems like Compton telescopes.

In Figure 2 we present the leakage current vs. temperature for detector 8911. The measurement of the leakage current of the center contact was performed with a pico ammeter. For this measurement, detector 8911 was biased at $1000 \mathrm{~V}$ and the guard ring was grounded. The leakage current is about a factor of 3 higher than that of a standard guard ring detector [8]. The I-V characteristic between the guard ring and the center contact was determined by biasing the guard ring in the range of $0 \mathrm{~V}$ to $1.0 \mathrm{~V}$ and measuring the current on the center contact. Based on the I-V characteristic, we have determined the electrical resistance of the region between the guard ring and the center contact of the detector. The resistance increases with the bias voltage across the detector and decreases with the temperature (Figure 3).

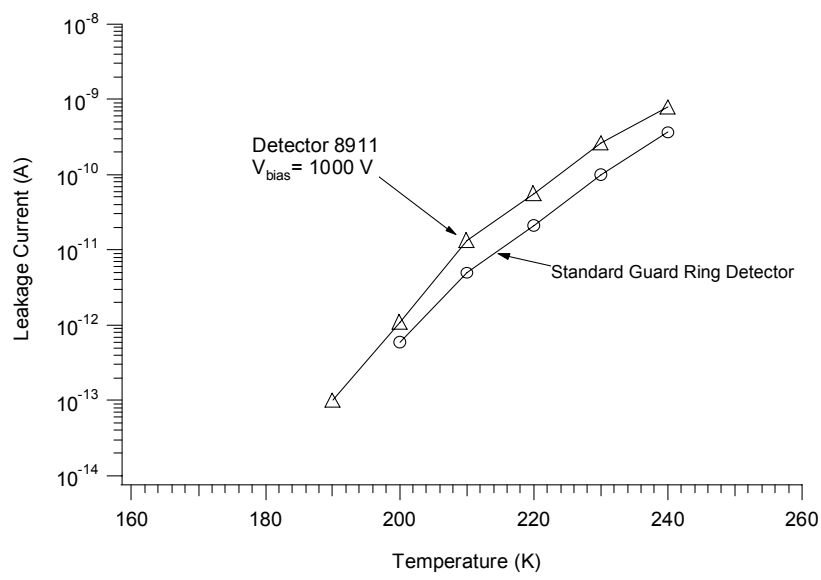

Figure 2. Leakage current versus temperature for the detector with $\alpha$-Si contact (detector 8911) measured at a bias voltage of $1000 \mathrm{~V}$. For comparison, the leakage current of a standard guard ring detector is also shown. The measurements were made with the guard ring grounded.

Based on this result we suppose that a pinch off of the surface charge region existing in the gap between the guard ring and the central contact takes place due to depletion at higher electric fields. A 50 -fold increase in the resistance is obtained when the electric field is increased by a factor of 2 . Even at a bias voltage of 1000 $\mathrm{V}$, the resistance obtained above $220 \mathrm{~K}$ is only on the order of a few hundreds of $M \Omega$. This is about three orders of magnitude lower than for detector 8905 , which had a 1 $\mathrm{mm}$ deep groove physically cut on its lithium-diffused contact to separate the guard ring and center contact [8]. The lower resistance obtained with the $\alpha$-Si contact is probably not a result of simple conduction within the $\alpha-\mathrm{Si}$ layer that is present in the gap region. The bias dependent of the resistance implies that interface states at the $\alpha-\mathrm{Si}$ /crystalline Si junction may be responsible. Further work is needed to understand the conduction mechanism and to increase the isolation resistance.

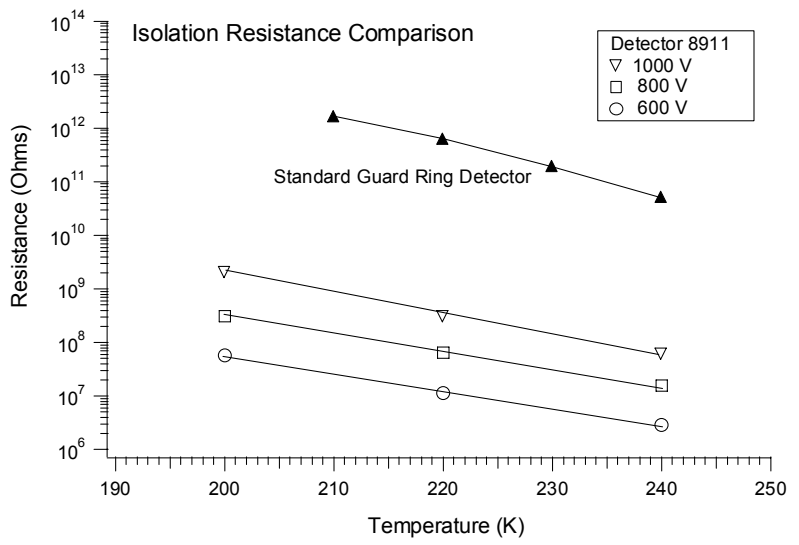

Figure 3. Guard ring isolation resistance vs. temperature in detector 8911. The isolation resistance increases with the bias voltage applied on the detector. A standard guard ring detector [8] has an isolation resistance about three orders of magnitude higher than the n-type contact obtained with $\alpha-\mathrm{Si}$.

The spectrometric performance of detector 8911 is presented in Figure 4. The FWHM obtained at a temperature of $240 \mathrm{~K}$ for the ${ }^{57} \mathrm{Co} 122 \mathrm{keV}$ line is about $2.57 \mathrm{keV}$. The variation of the FWHM with temperature is shown in Figure 5. The major increase of the FWHM with temperature is manifested above $200 \mathrm{~K}$ due to the increase in the leakage current.

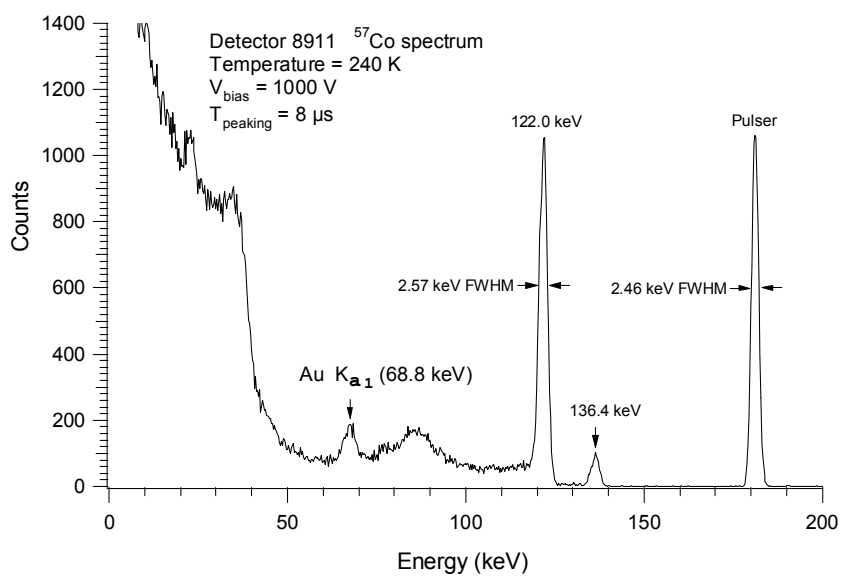

Figure $4 .{ }^{57} \mathrm{Co}$ spectrum obtained with detector 8911 at $240 \mathrm{~K}$ and 1000 $\mathrm{V}$ bias voltage. A FWHM of $2.57 \mathrm{keV}$ was obtained for the $122 \mathrm{keV}$ peak. 


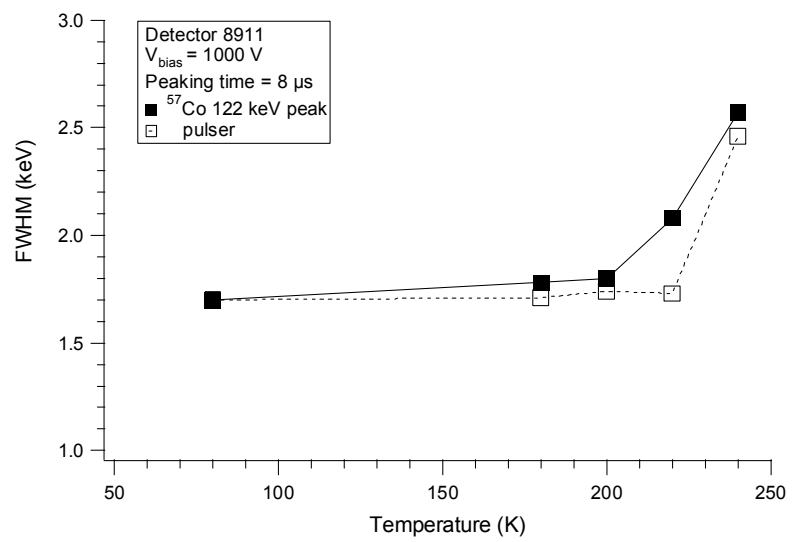

Figure 5. FWHM vs.Temperature for detector 8911. FWHM values were obtained from ${ }^{57} \mathrm{Co}$ spectra at a bias voltage of $1000 \mathrm{~V}$. The major increase in FWHM is manifested above $200 \mathrm{~K}$.

\section{Development of new P-type Contact}

While the $\alpha-\mathrm{Si}$ contacts work well as replacement of the lithium contacts, they exhibit large leakage currents when used as p-type contacts. Therefore, a different contact needed to be developed to replace the gold surface barriers. Most processes used to form p-type contacts use boron implantation followed by annealing at relatively high temperatures, e.g. $900{ }^{\circ} \mathrm{C}$, to activate the boron and to anneal out the lattice damage. However, previous experiments shown that thermal cycling of the float zone silicon material used to make $\mathrm{Si}(\mathrm{Li})$ detectors to $900^{\circ} \mathrm{C}$ results in severe degradation of the detector performance. Therefore we first investigate the highest annealing temperature which can be used, and then develop the optimal process for producing a boronimplanted contact. In any case, the thermal annealing required for the boron contact, means that the contact formation needs to be performed prior to lithium drifting.

\subsection{Thermal annealing experiments}

In order to find the optimum annealing temperature to activate the boron implant, we have thermally annealed two different sets of $3 \mathrm{~mm}$ thick silicon wafers (with no boron implantation) at temperatures between $500^{\circ} \mathrm{C}$ and $650^{\circ} \mathrm{C}$, in $50^{\circ} \mathrm{C}$ steps. After annealing, full area $\mathrm{Si}(\mathrm{Li})$ detectors were fabricated using these wafers, with the regular lithium n-type contact and gold barrier p-type contact. For both sets of detectors, no significant changes in the electronic properties are noticed for the detectors annealed up to $550^{\circ} \mathrm{C}$. The most significant changes have only been observed for the detectors annealed at $600^{\circ} \mathrm{C}$ and $650^{\circ} \mathrm{C}$.

The most important aspect noticed for the detectors annealed at $600^{\circ} \mathrm{C}$ and $650^{\circ} \mathrm{C}$ resides in the charge collection and spectra formation. Above approximately $180 \mathrm{~K}$, it is not possible to get a high quality gamma-ray spectrum with the detectors that have been annealed at $600^{\circ} \mathrm{C}$ and $650^{\circ} \mathrm{C}$ by applying the nominal bias voltage of $400 \mathrm{~V}$. For example, with detector 8944 (annealed at $600^{\circ} \mathrm{C}$ ) at $400 \mathrm{~V}$ bias $200 \mathrm{~K}$, the 122 $\mathrm{keV}$ line of ${ }^{57} \mathrm{Co}$ is no longer normally formed on the spectrum, although it was normal at $180 \mathrm{~K}$. This behavior is noticed for the detectors thermally annealed at $600^{\circ} \mathrm{C}$ and $650^{\circ} \mathrm{C}$ only. Detectors annealed at $550^{\circ} \mathrm{C}$ showed no degradation of the spectra (Figure 6).

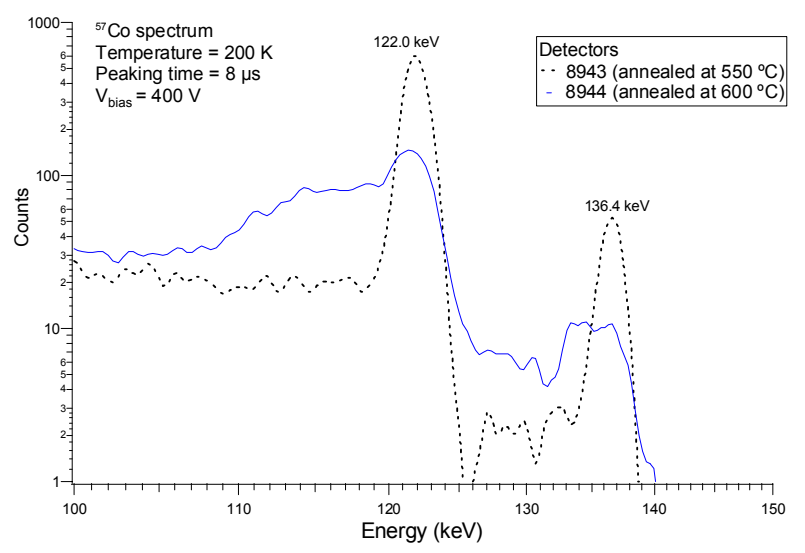

Figure $6 .{ }^{57} \mathrm{Co}$ spectra achieved with detector 8943 (annealed at $550{ }^{\circ} \mathrm{C}$ ) and 8944 (annealed at $600{ }^{\circ} \mathrm{C}$ ). Detector 8944 shows a poor charge collection and a deformed spectrum compared to detector 8943 .

Our hypothesis is that the poor charge collection which leads to distorted spectra is due to the existence of deep levels in silicon acting as traps that impedes charge collection. For the second set of annealing experiments, we carried out the thermal annealing in a facility that is used for making high-resistivity silicon detectors with ultra-low leakage current $\left(\sim 100 \mathrm{pA} / \mathrm{cm}^{2}\right.$ at $\left.296 \mathrm{~K}\right)$. The results are the same, which indicates that the defects responsible for the poor detector performance with the high temperature annealing are likely not due to externally introduced contamination.

Based on these results we can conclude that the properties of the material that is annealed at temperatures above $600^{\circ} \mathrm{C}$ have been changed so as the charge collection is affected. However, the degradation is much less severe at annealing temperatures under $550^{\circ} \mathrm{C}$, as shown in Figure 6.

\subsection{Boron implanted contacts}

To fabricate a detector with boron-implanted contacts, a $2 \mathrm{~mm}$ thick silicon wafer is first implanted with boron on one side. The wafer is implanted cold, at a temperature of $110 \mathrm{~K}$ in order to obtain a higher level of electrical activation of the boron [9]. After implantation, the wafer was annealed in nitrogen gas at a temperature of $500^{\circ} \mathrm{C}$ in order to activate the boron implant. The detector is then fabricated in a full area configuration by using the standard $\mathrm{Si}(\mathrm{Li})$ process, having a standard lithium contact as the n-type contact. A layer of 500 
Angstrom of aluminum is evaporated on the boronimplanted contact. The detector is then modified into a guard ring structure by using a masking process (Figure $1)$. The areas of the guard ring and the center contact were covered with an etch-resistant mask. The annular region that remained exposed was chemically etched in order to form a gap between the two contacts. The leakage current presented in Figure 7 shows values comparable to or lower to the standard guard ring detector 8905 [8].

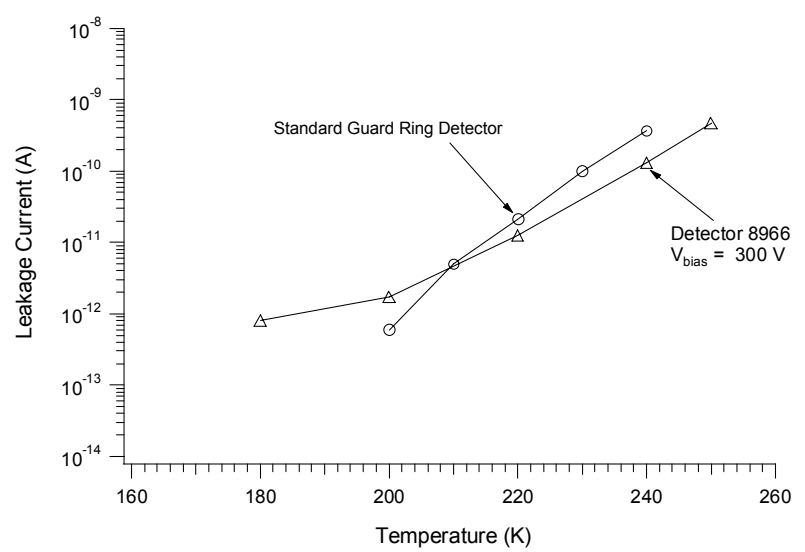

Figure 7. Leakage Current of detector 8966 at a bias voltage of $300 \mathrm{~V}$. For comparison, the leakage current of a standard guard ring detector is also shown. The measurements were made with the guard ring grounded.

When the bias voltage across the detector is increased, the isolation resistance between the guard ring and the central region increases (Figure 8). The resistance determined at $240 \mathrm{~K}$ is on the order of $10^{9} \Omega$. In this case, for the boron implanted p-type contact, the isolation resistance is high enough to ensure a good separation between the strips even at temperatures around $240 \mathrm{~K}$. With detector 8966 at $240 \mathrm{~K}$, we have obtained a ${ }^{57} \mathrm{Co}$ spectrum with a FWHM of $2.54 \mathrm{keV}$ for the $122 \mathrm{keV}$ line (Figure 9). The variation of the FWHM with temperature for detector 8966 is shown in Figure 10. Similarly to detector 8911 and the standard detectors in [8], the most significant increase of FWHM is manifested above 200 K.

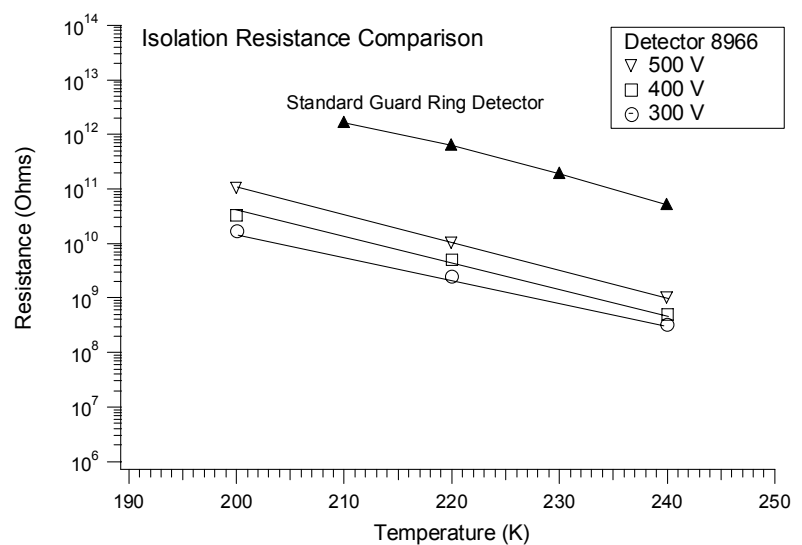

Figure 8. Guard ring isolation resistance in detector 8966. The minimum resistance is on the order of $10^{9} \Omega$ at $240 \mathrm{~K}$. An increase of the resistance with bias voltage is apparent.

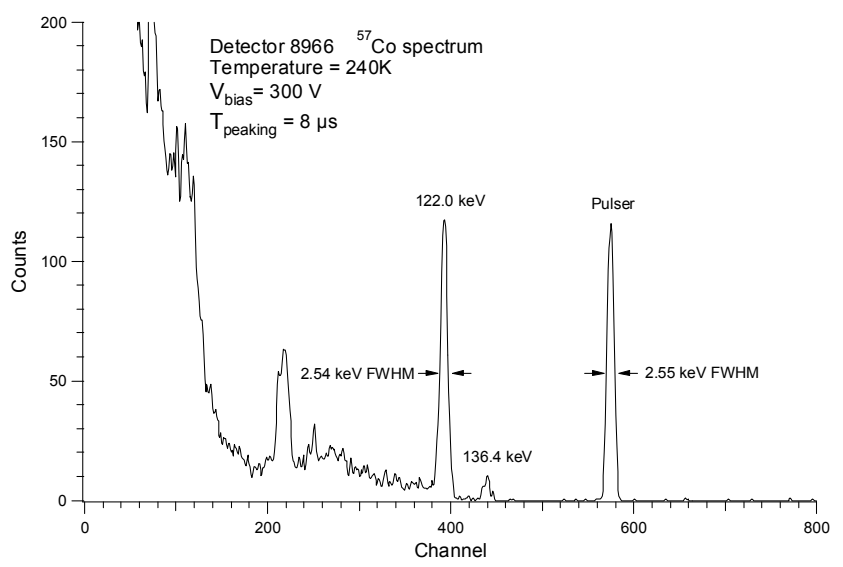

Figure $9 .{ }^{57} \mathrm{Co}$ spectrum obtained with detector 8966 at $240 \mathrm{~K}$ and a bias voltage of $300 \mathrm{~V}$. A FWHM of $2.54 \mathrm{keV}$ was obtained for the $122 \mathrm{keV}$ peak.

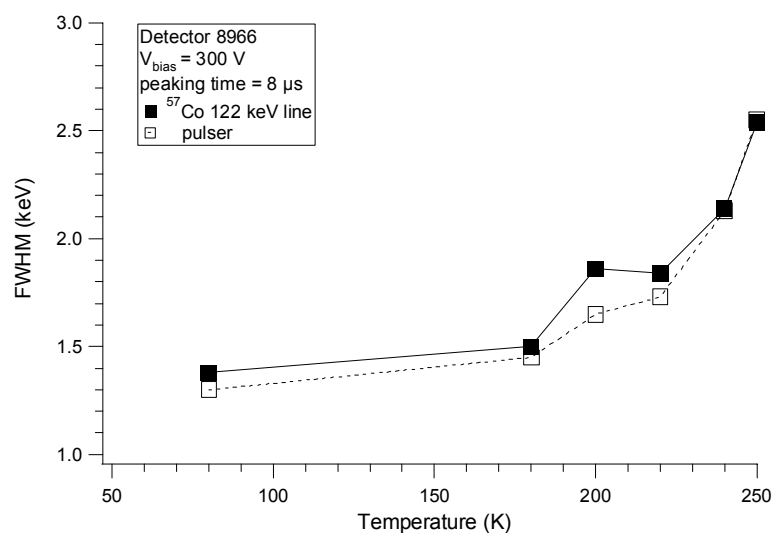

Figure 10. FWHM vs. Temperature for detector 8966. FWHM values were obtained from ${ }^{57} \mathrm{Co}$ spectra at a bias voltage of $300 \mathrm{~V}$. 


\section{Conclusions}

The experimental results show that it is possible to use with good spectral performance amorphous silicon $(\alpha-\mathrm{Si})$ as the n-type contact and boron implanted silicon as the p-type contact for $\mathrm{Si}(\mathrm{Li})$ detectors operated at temperatures up to $240 \mathrm{~K}$. The n-type contact obtained by depositing $\alpha-\mathrm{Si}$ on crystalline silicon provided encouraging results although further work should be performed in order to increase the isolation resistance between contact elements. The boron implanted p-type contact provided good results for the detectors implanted at temperatures around $110 \mathrm{~K}$ and thermally annealed at temperatures of maximum $550^{\circ} \mathrm{C}$. Detectors thermally annealed at temperatures above $600^{\circ} \mathrm{C}$ have shown a degradation in performance when operated above $200 \mathrm{~K}$. For both the $\alpha$-Si n-type contact and boron implanted ptype contact, the isolation resistance between contact elements increased substantially as detector bias voltage increased. At a detector bias of $1000 \mathrm{~V}$, the isolation resistance obtained for the $\alpha$-Si contact is on the order of $10^{8} \Omega$ at $240 \mathrm{~K}$. At a detector bias of $500 \mathrm{~V}$, the isolation resistance obtained for the boron-implanted contact is on the order of $10^{9} \Omega$ at $240 \mathrm{~K}$. We have also shown that the target spectrometric performance of $2 \mathrm{keV}$ FWHM could be achieved in the temperature range from $200 \mathrm{~K}$ to $240 \mathrm{~K}$. By operating the detectors at a temperature of $240 \mathrm{~K}$, both types of new contacts provided about $2.5 \mathrm{keV}$ FWHM when measuring the $122 \mathrm{keV}$ line of ${ }^{57} \mathrm{Co}$. Further work will be done in order to characterize a prototype strip detector having the contacts developed according to the process presented in this paper.

\section{Acknowledgements}

This work was supported by the Defense Threat Reduction Agency, Department of Defense, and the National Aeronautics and Space Administration, through the U.S. Department of Energy under Contract No. DEAC03-76SF00098.

\section{References}

[1] J.D. Kurfess, W.N. Johnson, R.A. Kroeger, and B.F. Phlips, 5th Compton Symposium, AIP Conference Proceedings, Volume 510, Issue 1, pp. $789-793$ (2000).

[2] M. Amman, P.N. Luke - "Position Sensitive germanium detectors for gamma-ray imaging and spectroscopy" - Proceedings of SPIE, Volume 4141, pp. 144-156 (2000)

[3] E. A. Wulf, J. Ampe, W. N. Johnson, R. A. Kroeger, J. D. Kurfess and B. F. Phlips "Depth Measurement in a Strip Detector", Proceedings of 2001 IEEE Nuclear Science Symposium, 410 Nov 2001, San Diego, CA
[4] D.A. Landis; Y.K. Wong; J.T. Walton; F.S. Goulding, IEEE Transactions on Nuclear Science -36, p.185 (1989).

[5] A. Fong, J.T. Walton, E.E. Haller, H.A. Sommer, and J. Guldberg, Nuclear Instruments and Methods, 199, p. 623 (1982).

[6] R.A. Street, "Hydrogenated Amorphous Silicon", Cambridge University Press, New York 1991, and references therein

[7] J. T. Walton, W.S. Hong, P.N. Luke, N.W. Wang, et al., "Amorphous silicon/crystalline silicon heterojunctions for nuclear radiation detection applications" - IEEE Trans. Nucl. Sci. vol. 44 (no. 3, pt. 1), (1996 Nuclear Science Symposium and Medical Imaging Conference NSS/MIC, Anaheim CA, USA, 3-9 Nov 1996), IEEE, June 1997 p. 961-4

[8] C. Tindall, I.D. Hau, P.N. Luke - "Evaluation of $\mathrm{Si}(\mathrm{Li})$ Detectors for Use in an Advanced Compton Telescope" - paper submitted to the $10^{\text {th }}$ Symposium on Radiation Detection and Application, University of Michigan Ann Arbor, May 21-23, 2002

[9] S.M. Sze, "VLSI Technology", McGraw-Hill, New York 1983 